\title{
PREPARATION OF CARBOXYMETHYL CELLULOSE/ACRYLAMIDE COPOLY-MER HYDROGEL USING GAMMA RADIATION AND INVESTIGATION OF ITS SWELLING BEHAVIOR
}

\author{
S. SULTANA ${ }^{1}$, M. R. ISLAM* ${ }^{2}$, N. C. DAFADER ${ }^{1}$ AND M. E. HAQUE ${ }^{1}$ \\ ${ }^{1}$ Nuclear and Radiation Chemistry Division (NRCD), Institute of Nuclear Science and Technology \\ (INST), Bangladesh Atomic Energy Commission (BAEC), G. P. Box-3787, Dhaka. ${ }^{2}$ Department of \\ Chemistry, Jahangirnagar University, Savar, Dhaka-1342, Bangladesh.
}

\begin{abstract}
Acrylamide (AAm)/carboxymethyl cellulose (CMC) blend hydrogels have been prepared by the application of gamma radiation from $\mathrm{C} 0-60$ source at room temperature $\left(27^{\circ} \mathrm{C}\right)$. The preparation conditions such as effect of CMC concentration and radiation dose on gel fraction and swelling behaviors were investigated. The maximum value of gel fraction is obtained at $25 \mathrm{kGy}$ radiation dose. The swelling properties were investigated in distilled water, saline solution $(\mathrm{NaCl})$ and buffer medium. The maximum swelling value of hydrogel was obtained at 48 hours in water. The swelling ratio of hydrogel decreased with increase in concentration of $\mathrm{NaCl}$ in swelling medium. In buffer, it was found that swelling ratio increased with increase in $\mathrm{pH}$ of medium.
\end{abstract}

\section{Introduction}

Increasing interest in natural-based super absorbent hydrogel has developed mainly due to high hydrophilicity, biocompatibility, non-toxicity, and biodegradability of biopolymers. These materials are defined as cross-linker macromolecular networks that can absorb water or physical fluids up to many times of their own weight in a short time, but are not dissolved when brought into contact with water ${ }^{\mathbf{1}}$. Because of excellent characteristics, superabsorbent hydrogels are widely used in many fields, such as agricultural and horticultural, disposable diapers, feminine napkins, pharmaceuticals and medical applications ${ }^{2-3}$. Polysaccharides such as starch, chitin/chitosan and their watersoluble derivatives have a variety of application in many fields owning to their unique structure, distinctive properties, safety and biodegradability ${ }^{4}$. Cellulose is one of the most abundant naturally occurring polymers. It is commonly found in the cell walls of plants and certain algae. Some bacteria (e.g., Acetobacter xylinum) are also able to synthesize cellulose $^{5}$. Carboxymethyl cellulose (CMC) is an anionic water soluble natural polymer derivative, which is biocompatible, renewable, and non-toxic polysaccharides ${ }^{6-8}$. CMC formed hydrogel by the action of ionizing radiation at high concentration in aqueous solution $^{9}$. It has been reported that a novel biodegradable hydrogel of CMC was synthesized without any additives from paste like solution of CMC in water by the application of radiation ${ }^{10}$. Wach et al. ${ }^{11}$ studied the effect of radiation dose and irradiation atmosphere on the properties of CMC hydrogel. They found that gel fraction of CMC in vacuum atmosphere was higher than that of CMC in air atmosphere. In hydrochloric acid solution (0.5M), CMC formed physical gel. FTIR spectra indicated that sodium in CMC is replaced by hydrogen in acid solution ${ }^{12}$. Acrylamide is used for the production of high molecular weight polyacrylamides, which are modified to produce different physical and

*Author for Correspondence; e-mail: rabiulju@gmail.com 
chemical properties suited to a wide variety of industrial applications. Poly(acrylamide) (PAAm) hydrogels is highly hydrophilic, neutral and inert materials. PAAm hydrogels have sufficient hydrophilicity, but are low hydrolytic stability and tensile strength. Hydrogels derived from PAAm have many useful chemical and physical properties and, consequently, have been investigated for applications as smart polymers ${ }^{13}$. In our earlier work, it has observed that addition of salts $\left(\mathrm{CaCl}_{2}\right.$ and $\left.\mathrm{NaCl}\right)$ have a significant effect on cross-linking of CMC solution under irradiation ${ }^{14}$. It is also observed that CMC with $\mathrm{CaCl}_{2}$ gives a good gel value than that of $\mathrm{CMC}$ with $\mathrm{NaCl}$. The maximum gel value of $\mathrm{CMC}$ hydrogel was achieved at $0.2 \mathrm{M}$ ionic strength of $\mathrm{CaCl}_{2}$. The present work was undertaken to prepare hydrogel from AAm/CMC blends by the application of gamma radiation and effect of CMC concentration and irradiation dose on swelling properties in distilled water, saline solution and different $\mathrm{pH}$ mediums were discussed.

\section{Experimental}

\section{Raw Materials}

Acrylamide was purchased from Aldrich Chemical and purity was 97\%. CMC was purchased from Sigma; $\mathrm{NaCl}$ was purchased from Fluka, Switzerland.

\section{Preparation of Acrylamide/CMC co-polymer Hydrogel}

Homogeneous solutions containing 5\% of acrylamide were prepared in deionized water with different concentration of CMC (2, 3 \& 5\%). Then the solutions were poured in several test tubes, sealed with polyethylene and finally irradiated with gamma-rays from Co-60 source by varying radiation dose of 10, 15, 20, 25, 30 and $35 \mathrm{kGy}$ at room temperature $\left(27^{\circ} \mathrm{C}\right)$. The irradiated samples were cut into small pieces, dried at room temperature and then in an oven at $50^{\circ} \mathrm{C}$ to constant weight for measurement of gel fraction and swelling properties.

\section{Measurement of Gel Fraction}

Prepared sample was immersed in deionized water for 48 hours at room temperature for removing sol parts (soluble portion). Then the undissolved part of sample was taken out, dried in air and finally dried in a vacuum oven at $50^{\circ} \mathrm{C}$ to constant weight. The gel fraction was calculated as follows:

Gel fraction $(\%)=\left(\mathrm{W}_{\mathrm{d}} / \mathrm{W}_{\mathrm{i}}\right) \times 100$

Where, $W_{i}$ is the initial weight of dried gel sample and $W_{d}$ is the weight of dried gel sample after extraction in deionized water.

\section{Measurement of Swelling Ratio}

The dried gel sample (extracted in water) was immersed in deionized water and other test solutions ( $\mathrm{NaCl}$ solution and $\mathrm{pH}$ medium) for 48 hours at room temperature; the hydrogel was filtered using a stainless steel net of 30 meshes and weighed after removing the surface water by soft tissue paper. The swelling ratio was calculated as follows: 
Swelling ratio $\left.=\left[\mathrm{W}_{\mathrm{s}}-\mathrm{W}_{\mathrm{i}}\right) / \mathrm{W}_{\mathrm{i}}\right]$,

Where, $\mathrm{W}_{\mathrm{s}}$ is the weight of gel in swollen state and $\mathrm{W}_{\mathrm{i}}$ is the initial weight of water extracted dry gel sample

\section{Measurement of Water Absorption}

The dried gel sample (extracted in water) was immersed in distilled water for different time at room temperature. At first day weight of the samples were taken one hour interval, 6 hours interval at the second, third and fourth days. The Water Absorption was calculated as follows:

Water Absorption $\left.(\%)=\left(\mathrm{W}_{\mathrm{t}}-\mathrm{W}_{\mathrm{i}}\right) / \mathrm{W}_{\mathrm{i}}\right] \times 100$

Where, $W_{t}$ is the weight of gel in swollen state at time $t$ and $W_{i}$ is the initial weight of water extracted dry gel sample.

\section{Results and Discussion}

\section{Gel Fraction}

Fig.1 shows the effect of radiation dose and concentration of AAm/CMC on the gel fraction of AAm/CMC grafted co-polymer hydrogel. It is found that gel fraction increases with increased radiation dose and attains maximum at the radiation dose of $25 \mathrm{kGy}$. After that radiation the value of gel content is almost same. It is also found that gel fraction depends on the concentration of CMC.

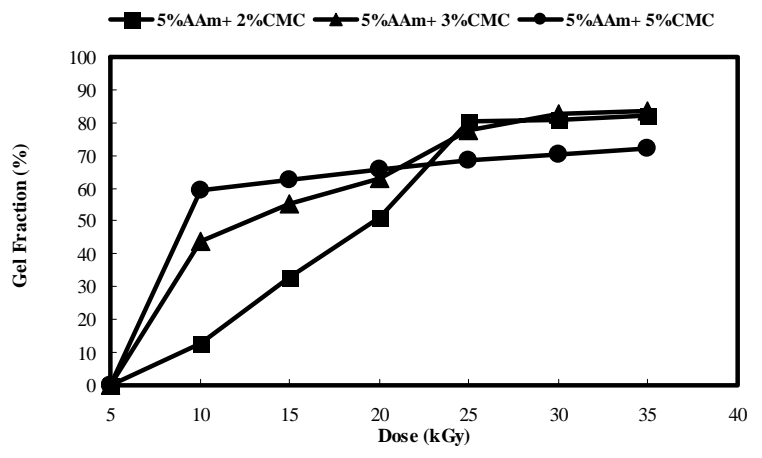

Fig. 1: Effect of radiation dose and concentration of $\mathrm{CMC}$ on gel fraction of Acrylamide /CMC blend hydrogels

\section{Swelling Ratio in distilled water}

Fig.2 shows the effect of radiation dose on swelling ratio of different concentration of CMC with 5\% AAm co-polymer hydrogel. It is found that swelling ratio decreases with increased radiation dose. The decreasing trend of radiation dose is fast up to $25 \mathrm{kGy}$, after that it becomes slow. It is also found that swelling ratio depends on the concentration of CMC. AAm with $2 \%$ CMC give the higher swelling value. 


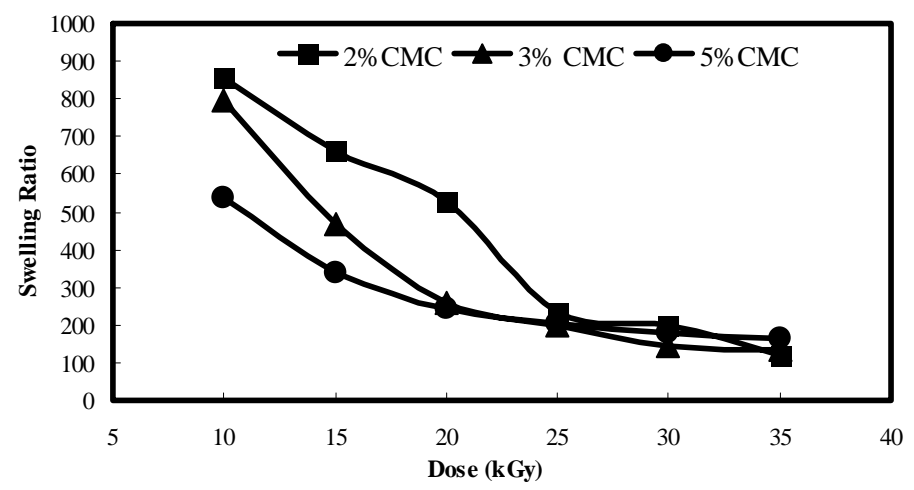

Fig.2: Effect of radiation dose and concentration of CMC on Swelling Ratio of 5\%Acryl Amide/CMCblend hydrogel in deionized water

Swelling Ratio in $\mathrm{NaCl}$ solution

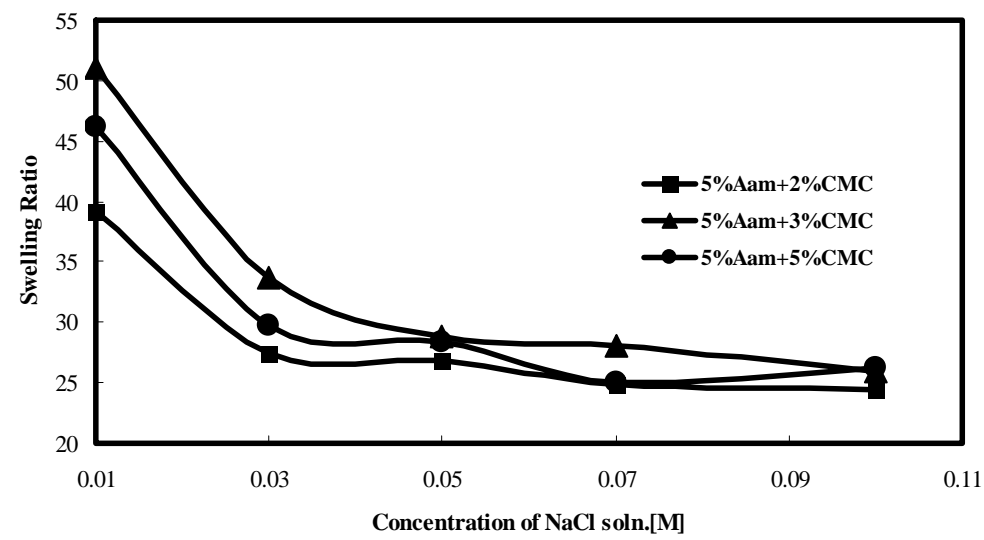

Fig. 3: Effect of concentration of CMC on swelling ratio of AAm/CMC grafted co-polymer hydrogel in $\mathrm{NaCl}$ solution at $25 \mathrm{kGy}$ [Standing time $48 \mathrm{~h}$ ]

Fig.3 describes the response in swelling to the ionic strength of solution of the three different concentration of CMC with AAm co-polymer hydrogel (2, 3 and 5\% CMC) at $25 \mathrm{kGy}$. From figure it is found that the swelling ratio decreases with increasing the ionic strength of $\mathrm{NaCl}$ solution. This may be explained that at low ionic strengths, the concentration of bund charges within the SWA network exceeds the concentration of salt in the external solutions; a large ion-swelling pressure causes the hydrogel to expand, thereby swelling ratio is higher than those of in higher concentration of ions. As the external salt concentration rises, the difference between the internal and external ion 
concentration decreases and the SWA deswells. The hydrogel continues to deswell with increasing external salt concentration until the mobile-ion concentrations inside and outside are approximately equal. This phenomenon can also be explained on the basis of repulsion between fixed charged groups on SWA. As ionic strength increases in swelling medium, repulsion is shielded and hydrogel deswelled.

\section{Swelling Ratio in different $\mathrm{pH}$ medium}

Fig.4 represents the effect of $\mathrm{pH}$ on the swelling ratio of AAm/CMC co-polymer hydrogel prepared at $25 \mathrm{kGy}$. It is found that swelling ratio increases with the increase in $\mathrm{pH}$ of swelling medium (i.e., from acidic region to basic region). The lower swelling in the more acidic region may be due to the protonation of the carboxylic group of CMC in SWA and thereby resisting the association with water through $\mathrm{H}$-bonding. With increased the $\mathrm{pH}$ of swelling medium de-protonation takes place and it is found that the maximum swelling value is obtained at $\mathrm{pH} 8$.

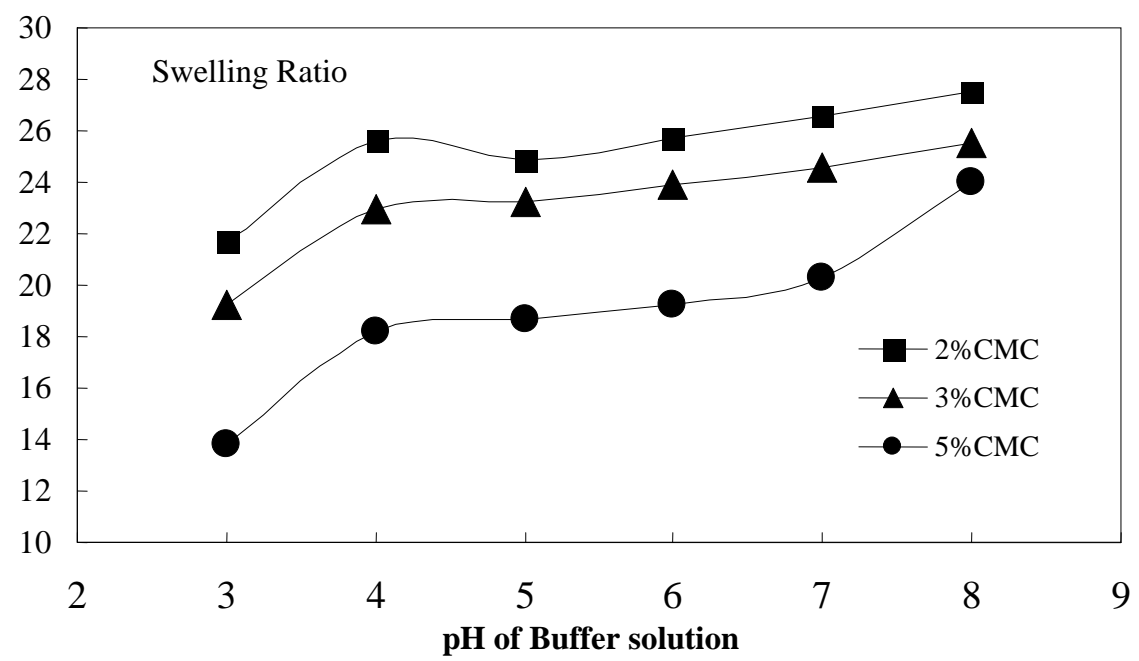

Fig. 4. Effect of concentration of CMC on swelling ratio of Am/CMC blend hydrogel in buffer solution at $25 \mathrm{kGy}$ [standing time $48 \mathrm{~h}$ ] 


\section{Water Absorption (\%)}

Fig. 5 shows the absorption of water of AAm/CMC co-polymer hydrogel. It is found that maximum water absorption occurs at 48 hours and after that the absorption of water decreases. It is also found that AAm with 3\% CMC give the highest water absorption value. It was found that after obtained maximum value of water absorption, part of the SWA is disintegrated. This may be the cause of reduced water absorption of SWA after 48 hours standing period.

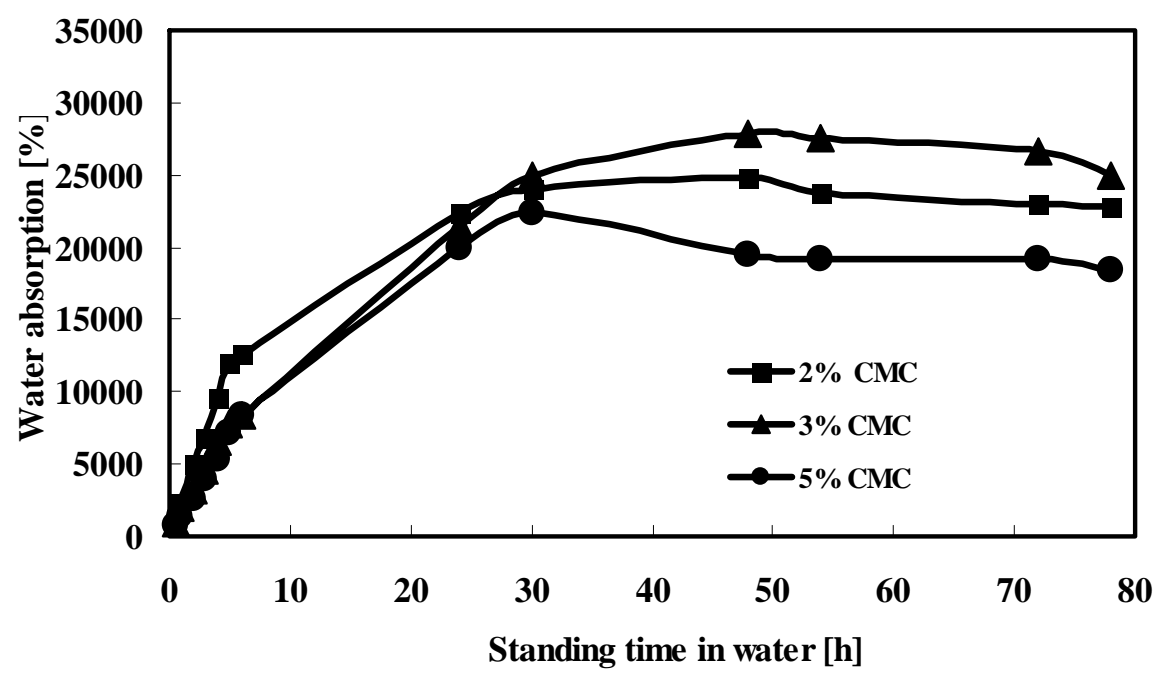

Fig.5: Effect of CMC on water absorption of AAm/CMC blend hydrogel prepared at $25 \mathrm{kGy}$

\section{Conclusion}

AAm/CMC copolymer hydrogel can be performed by the application of gamma irradiation from C0-60 source with different doses (10, 15, 20, 25, 30 \& 35kGy). The gel fraction of co-polymer hydrogel obtained maximum value at $25 \mathrm{kGy}$ radiation dose. Swelling ratio in distilled water decreases from 795 to 134 for copolymer hydrogel with 3\% CMC due to increased radiation dose from 10 to $35 \mathrm{kGy}$. The highest swelling ratio in distilled water is obtained from hydrogel with 3\% CMC. Swelling ratio of copolymer hydrogel decreases with increase in concentration of $\mathrm{NaCl}$ in swelling medium. With increase in $\mathrm{pH}$ of the swelling medium swelling ratio increases. Therefore, it can be concluded that AAm/CMC copolymer hydrogel in terms of swelling ratio in different swelling medium is environmental sensitive hydrogel. 


\section{References}

1. F. L. Buchholz and A. T. Graham, “Modern Superabsorbent PolymerTechnology”, Elsevier, Amsterdam, 1997.

2. L.B. Peppas and R.S. Harland, "In Absorbent Polymer Technology”, "Elsevier, Amsterdam , 1990.

3. R. Po, J. Macromol Sci. Rev. Macromol Chem. Phys., 1994 C 34, 607

4. S Dumitriu et al., "Polysaccharides in Medical Applications”, Marcel Dekker Inc, New York, 1996.

5. P. Ross, R. Mayer and M. Benziman, Microbiol Rev. 1991, 55 (1), 35058

6. B.Tosmic, B. Simoncic, B. Orel, A. Vilcnik and H. Spreizer, Carbohydrate Polymer, 2007, 69 (3), 478.

7. T. Miyamoto, S. Takahasi, H. Ito, H. Inagaki and Y. Nioshiki, J. Biomed. Mater. Res., 1989, 23(1), 125.

8. E. Entcheva, H. Bien, L. Yin, C. Y. Chung, M. Farrell and Y. Kostov, Biomaterials, 2004, 25 (26), 5753.

9. R. A. Wach, H. Mitomo, N. Nagasawa and F. Yoshii, Radiation Physics and Chemistry, 2003, 68 771-779.

10. B. Fei, R. A. Wach, H. Mitomo, F. Yoshii and T. Kume, J. Applied Polymer Science, 2000, 78 278-283.

11. R. A. Wach, H. Mitomo, F. Yushii and T. Kume, J Applied Polymer Science, 2001, 81, 3030-3037.

12. M. Takigami, H. Amada, N. Nagasawa, T. Yagi and M. Tamada, The Society of Fiber Science and Technology, Japan, 2006, 61, No. 3, p. 158 (Autumn Meeting).

13. W. Li, H. Zhao, P. R. Teasdale, R. John and S. Zhang, React. Funct. Polym., 2002, 52, 31.

14. S. Sultana, M. R. Islam, N. C. Dafader, M. E. Haque, N. Nagasawa and M Tamada, Int. J. Chem. Sci, 2012, 10(2), 627.

(Received : 25 May, 2012; Accepted : 29 November, 2012)

Fig. 1. Effect of radiation dose and concentration of CMC on gel fraction of Acrylamide /CMC blend hydrogels

Fig. 2. Effect of radiation dose and concentration of CMC on swelling ratio of $5 \%$ Acryl Amide/CMC blend hydrogel in deionized water

Fig. 3. Effect of concentration of CMC on swelling ratio of AAm/CMC grafted co-polymer hydrogel in $\mathrm{NaCl}$ solution at $25 \mathrm{k} \mathrm{Gy}$ [Standing time $48 \mathrm{~h}$ ]

Fig. 5. Effect of CMC on water absorption of AAm/CMC blend hydrogel prepared at 25kGy 